\title{
Exploring multivariate representations of indices along linear geographic features
}

\author{
Susanne Bleisch, ${ }^{\mathrm{a}}$ and Daria Hollenstein ${ }^{\mathrm{a}}$ \\ ${ }^{a}$ FHNW University of Applied Sciences and Arts Northwestern Switzerland, Institute of Geomatics Engineering, Muttenz, \\ Switzerland; susanne.bleisch@fhnw.ch,daria.hollenstein@fhnw.ch
}

\begin{abstract}
A study of the walkability of a Swiss town required finding suitable representations of multivariate geographical da-ta. The goal was to represent multiple indices of walkability concurrently and visualizing the data along the street network it relates to. Different indices of pedestrian friendliness were assessed for short street sections and then mapped to an overlaid grid. Basic and composite glyphs were designed using square- or triangle-areas to display one to four index values concurrently within the grid structure. Color was used to indicate different indices. Implement-ing visualizations for different combinations of index sets, we find that single values can be emphasized or de-emphasized by selecting the color scheme accordingly and that different color selections either allow perceiving single values or overall trends over the evaluated area. Values for up to four indices can be displayed in combination within the resulting geovisualizations and the underlying gridded road network references the data to its real world locations.
\end{abstract}

Keywords: Geovisualization, multivariate data, glyphs, walkability indices, linear geographic features, grid-based

\section{Introduction}

Multivariate data along linear geographic features may typically be represented through a combination of line color, line width or line style (e.g. Slocum, McMaster, Kessler, \& Howard, 2009). However, depending on the nature of the multivariate data and the chosen visual variables the representations might become visually complex, even confusing, especially when the linear features are of irregular length, including short segments, or are close together as may be characteristic of, for example, street network data in downtown areas.

In a project concerned with the analysis of the walkability of a Swiss town (Bachmann et al., 2016), we aimed at finding a suitable two-dimensional representation for a multivariate dataset of walkability indices along roads that allows for the concurrent visual analysis of several data dimensions. The representations should support the direct comparison of index values at single locations and across locations along the road network. The reminder of this paper describes and illustrates the derivation of the index data and considerations regarding different representation alternatives. It is concluded by a discussion of possible applications of the designed representations to different index data sets.

\section{Data and design}

\subsection{Data}

For a study of the walkability of a Swiss town with a particular focus on the active mobility of elderly woman (Bachmann et al., 2016), amongst other indicators, the pedestrian friendliness of the street network was assessed using area-wide geographical data and GIS. Various common indicators of street walkability (cf. Clifton, Livi Smith, \& Rodriguez, 2007) were included in the analysis, such as the steepness of roads, the presence of sidewalks, traffic noise and traffic flow, street lighting, visible greenery along the streets, the availability of benches to rest. For each of those indicators an index was designed, where the values range from zero to one and where higher values correspond to greater pedestrian friendliness in order to facilitate easier visual analysis of the resulting graphs.

The variables were assessed on regularly sized (ca. $15 \mathrm{~m}$ ) street network sections. The splitting of the street segments into smaller sections allows exploring variation in walkability at a high spatial resolution. Considering different representation alternatives (see section 2.2) showed that the options for visualizing several variables concurrently are limited when the variables should still be perceivable as being related to street sections. To achieve greater representation flexibility, we mapped the street sections to a regular grid (cf. Fig. 1). Where more than one street section contributes to a grid cell, the largest variable value was used. This mapping could be changed depending on the semantics and the intended use of a data set or geovisualization. 


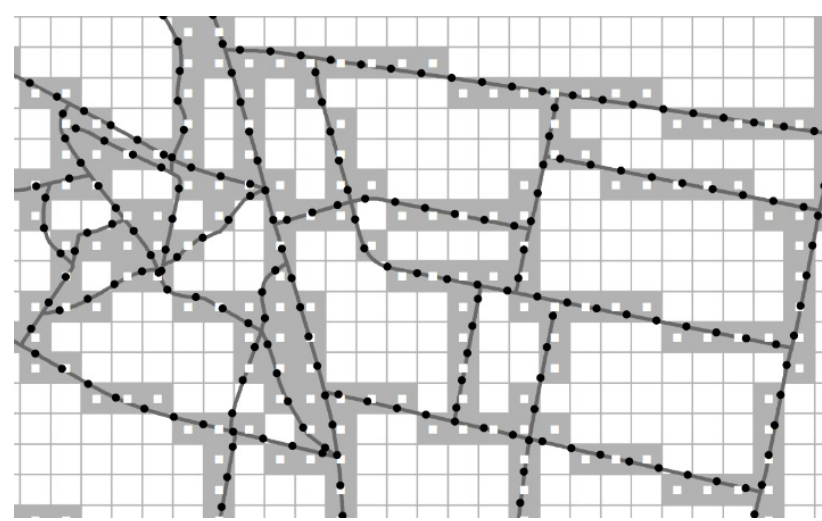

Fig. 1. Cropped illustration of the road network split-up in smaller sections (roads in black, dots indicating ends of sections) and overlaid grid. Grid cells in gray indicate cells where variable values from the road sections were assigned.

\subsection{Design considerations}

The variation of a single index along linear features can be represented either by varying color (cf. Fig. 2), line width or line style. However, considering the rather dense street network of a town with many short segments (see section 2.1) both, line width and line style, can be impractical as overlaps or difficult to discern value mappings may result.

Extending the approaches for linear representations of univariate data to multivariate displays poses additional challenges: We already considered line style and width to be potentially confusing for univariate data. A combination of those design elements for multivariate data seems even more difficult to interpret. Thus, we reformatted the index data in order to be able to produce representations that allow displaying up to four index values concurrently per street section without overlaps. As described in section 2.1 we produced a gridded data set from the original street network (cf. Fig 1). This way, the index values still basically vary along the linear road network, however, we are no longer required to actually use the line and apply line styles to display the data. With a gridded data set more options are available for representing multiple index values, for example, by designing a specific visual coding scheme using so-called glyphs (Borgo et al., 2013). Small glyph designs that fit within a single grid cell can be used to avoid overlap and arranging the glyphs along the linear features of the street network preserves an impression of the street network shape. Fig. 3 exemplifies the use of basic glyphs in a grid structure by showing univariate data through the use of a single proportional symbol, the areas of squares. The example shows the index values that re-late to the amount of greenery that is visible from the different street sections (Fig. 3). It seems possible to perceive the variation in values and concurrently the abstract road network from this figure.

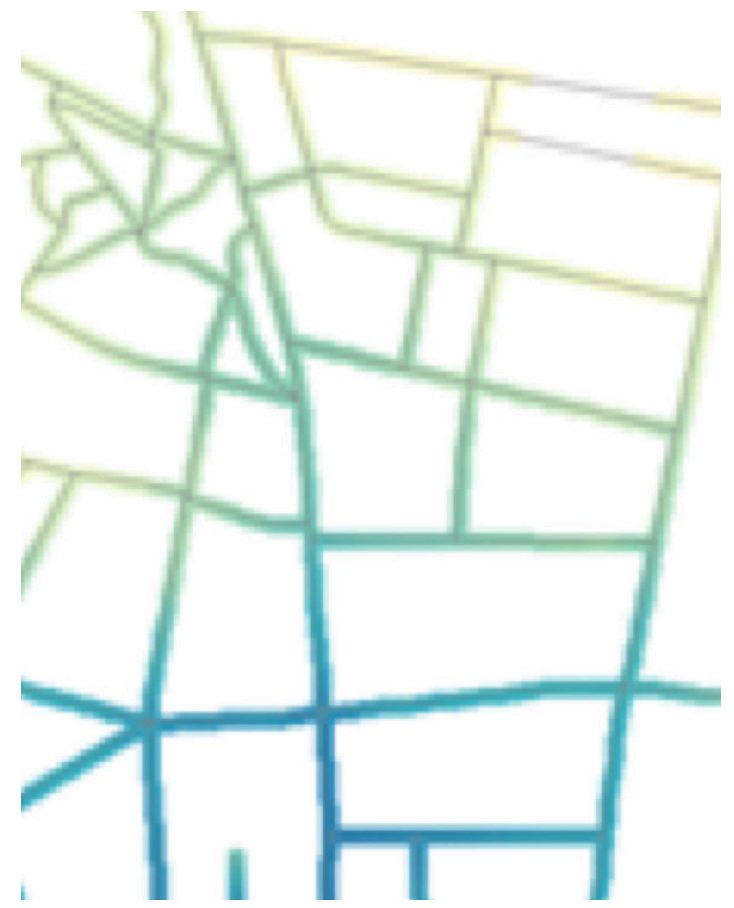

Fig. 2. Excerpt of a representation of the variation of a single index along a road network through color hues. Darker blueish color hues denote larger index values and lighter yellowish color hues denote smaller index values. (Source: Hollenstein \& Bleisch, 2016, p707)

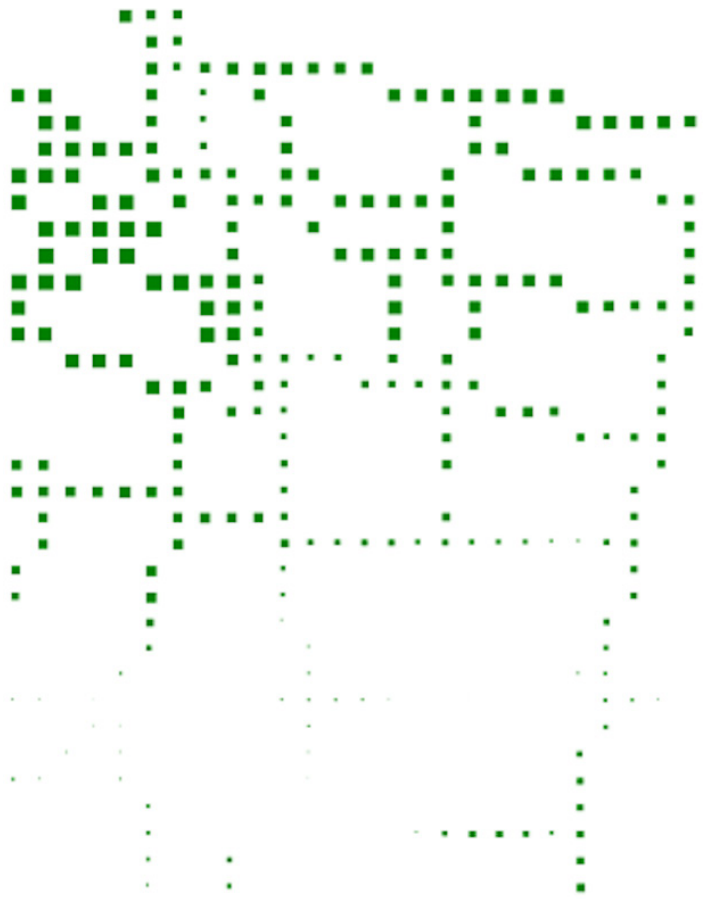

Fig. 3. Excerpt of the univariate representation of the index value 'greenery' through proportional symbols (areas of squares) along the road network. The variation of the values can be perceived concurrently with the abstracted street network layout.

Expanding the univariate representation to multivariate data requires the design of composite glyphs. For reasons of practicality and building on the grid-based structure, the composite glyphs accommodate up to four varia-ble values within a grid cell and make optimal use of the space available. Fig. 4 a) shows a possible 
subdivision of cell space (four cells with four areas to contain index value representations). Designs based on such a subdivision al-low for tight but non-overlapping arrangement of the different index value representations. Area is used to display the index values and color to show the index categories, thus employing the two most dominant visual channels ( $\mathrm{Li}$, Martens, \& Wijk, 2010). Typical area symbols are squares, triangles and circles. Circles were not used, as they do not make optimal use of the available space, leaving whitespace between circles. One index value is represented through a single square arranged centrally in the respective grid cell (Fig. 4 b). Two, three or four index values are represented as squares arranged in the subdivided grid cells (Fig. 4 a), c) and e). For three index values it seems ap-propriate to use areas of small triangles (Fig. $4 \mathrm{~d}$ ) as they make more balanced use of the available space.
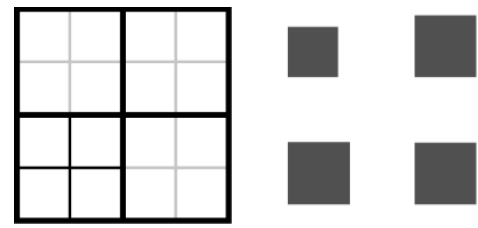

a)

b)
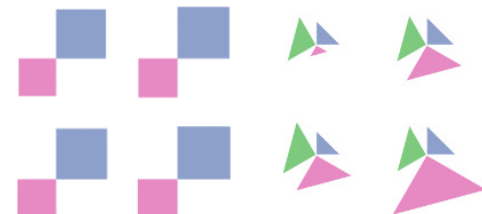

c)

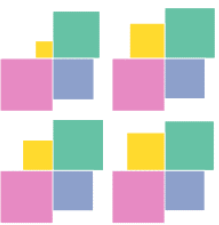

d)

e)

Fig 4 a) Four grid cells, each subdivided into four spaces to indicate the available space for up to four value representations; b) ex-ample of four single values represented through square areas each of which is located at the center of a grid cell (basic glyphs); c) ex-ample of four composite glyphs each showing two index values through diagonally arranged square areas; d example of four com-posite glyphs showing three index values through triangle areas; e) example of four composite glyphs showing four index values through square areas.

\section{Exemplary implementation and discussion of the glyph designs}

Different glyph designs were implemented for different combinations of indices. Regarding the choice of colors for the index categories different options emerged: Values can be emphasized or de-emphasized through the use of lighter or darker colors and respective combinations. Typically when choosing associative colors, for example yellow for the light index (Fig. 5 a), then the colors have different lightness values which, in addition to symbol size, influence the perception of the index values. Selecting colors with the same or very similar lightness values (Fig. 5 b) makes the single values more difficult to discern. However, especially in zoomed out views, the glyphs seem to merge and the focus is more on interpreting global patterns and trends, as no single color, and thus index, dominates the picture or dissipates in the background. Further research is needed to evaluate how efficient and effective those representation alternatives are in supporting value and pattern perception and decision-making.

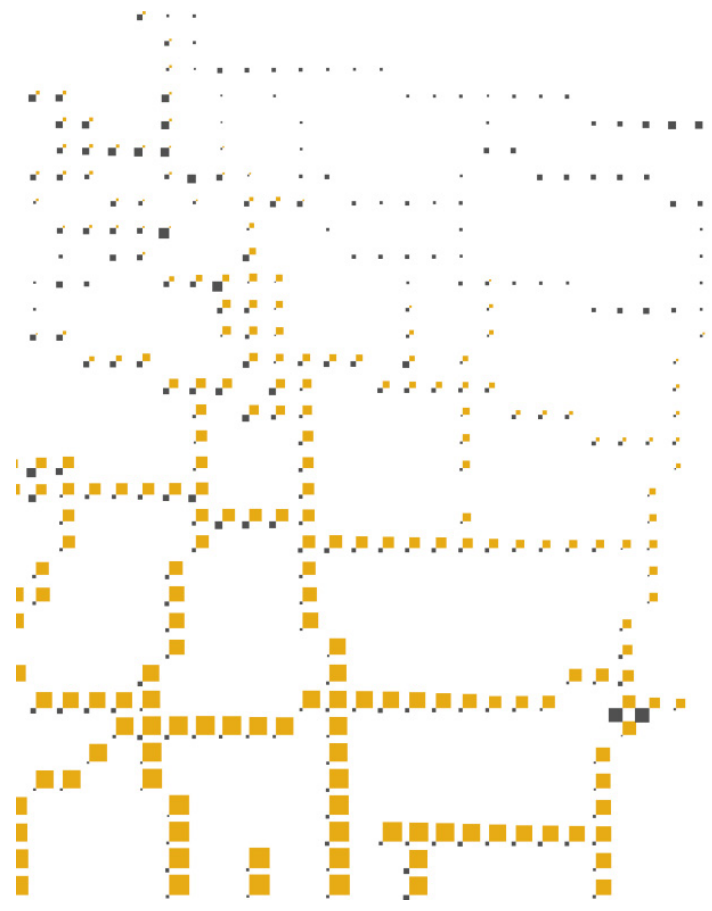

a)

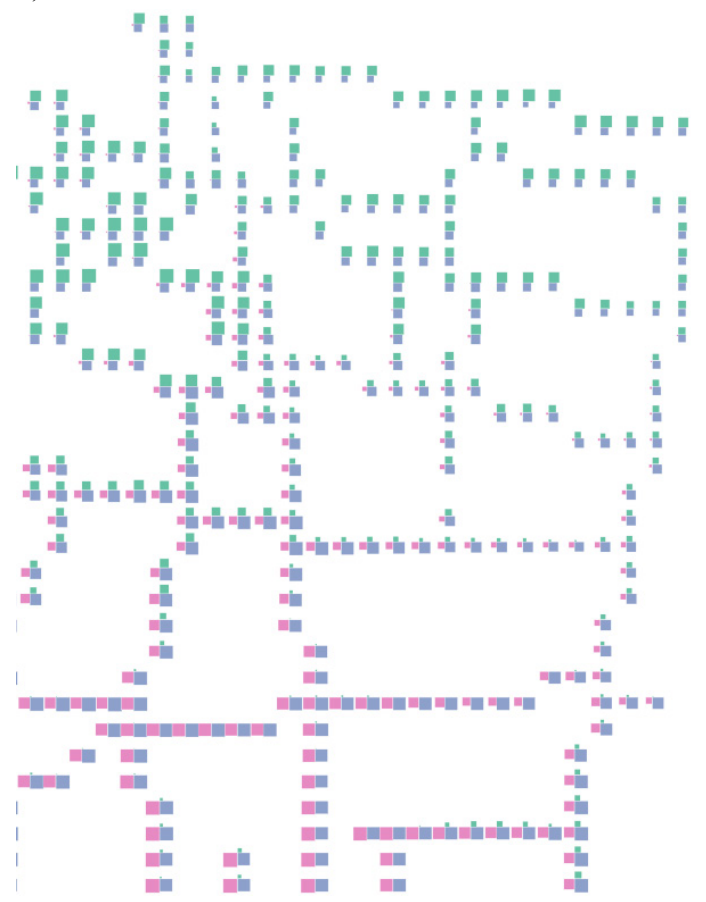

b)

Fig. 5. a) Excerpt of the road network with composite glyphs showing two indices using somewhat associative colors of differing lightness, the index showing street lighting in yellow and the slope index in black; b) excerpt of the road network with composite glyphs showing three indices using colors with very similar lightness values, shop accessibility pink, public transport accessibility blue and greenery green. 
Further, the composite glyph representation of the street network walkability can be combined with other spatial data. Fig. 6 a) and b) show the combination with subjective, survey based place assessments (Bachmann et al., 2016). Additionally, for non-densely packed glyph displays it might also be possible to add other background in-formation such as points of interest (for example, shops or other landmarks) that help orientation or are relevant for interpreting the displayed data.

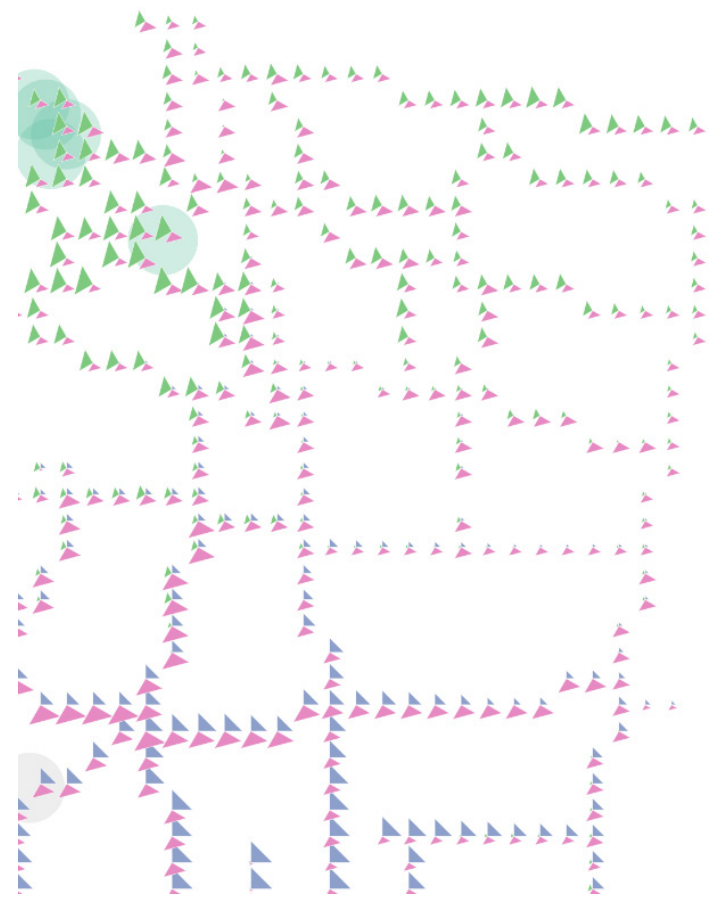

a)

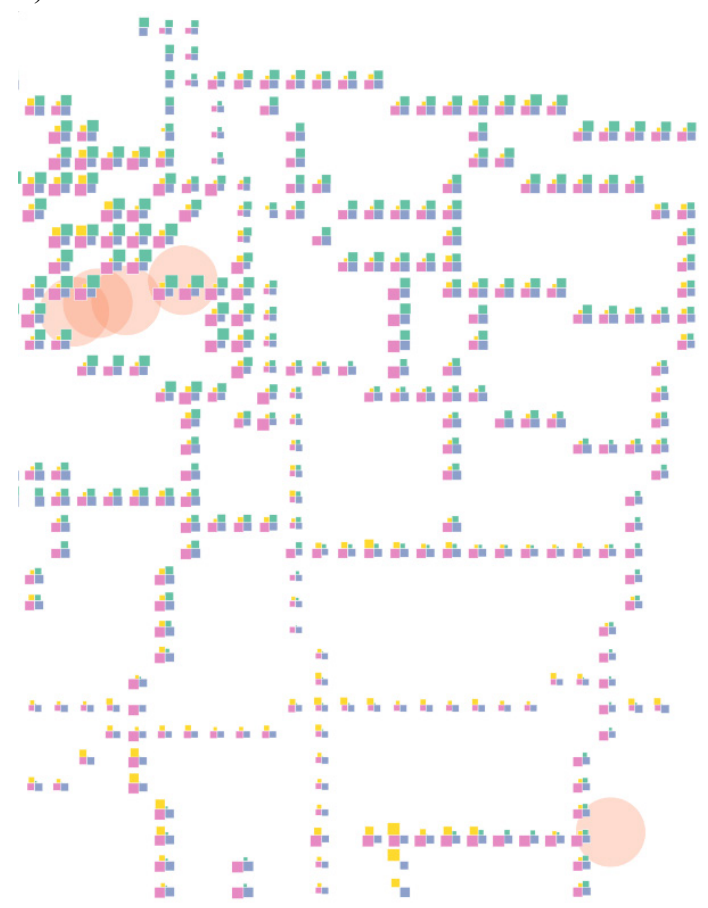

b)

Fig. 6. a) Excerpt of the road network with composite glyphs showing index values as triangle areas with some positive (green) and a neutral (grey) place indications in the background b) excerpt of the road network with composite glyphs showing index values as square areas with some negative (red) place indications in the background.

Using gridded data and working with indices, which all range from 0 to 1 , basically mitigates the problem of symbol overlap. However, there may still be areas where the glyphs are very small, and others where a few larger values, possibly outliers, dominate the representation. There are potentially areas within a dense street network where the composite glyphs are close to overlapping as they use up most of the grid cell space. It might be interesting to evaluate whether some degree of overlap may be tolerable, by for example using slightly bigger overlapping grid cells for the glyph placement, when the goal is seeing overall trends. In selected cases this may allow to emphasize very small glyphs, and potentially make them visible in the first place, when in other parts of the displays a few larger glyphs are allowed to overlap. First experiments have shown that especially when color values with similar lightness are chosen, some overlap, introduced by making the glyphs slightly bigger than the grid cells, seems not to hinder interpretation but this was not formally evaluated.

Informal discussions with project partners showed that the displays need some time to get used to. However, generally the gridded network design is appealing, as it is able to communicate the index values while still represent-ing the abstract road network, thus simplifying geographic referencing. The glyphs located along the gridded road network seem interpretable and when the road network is familiar, it is not too difficult to relate values to specific locations in the city. The mapping of the index values of the original street sections to the gridded network (cf. section 2.2) involves decisions, such as which cell size to choose or which values to assign when several values are mapped to the same grid cell. While different methods can be applied and argued for, those decisions also raise questions about the 'truthfulness' of the resulting geovisualization. Further research is needed to evaluate how well the resulting geovisualizations represent the underlying data, the readability and comparability of the representations and especially also the effectiveness with which relevant insight from combinations of walkability indices for city planning purposes may be gained.

\section{Acknowledgements}

The work presented here is part of and supported by the Strategic Initiative Ageing Society (Strategische Initiative Alternde Gesell-schaft) of the FHNW University of Applied Sciences and Arts Northwestern Switzerland (http://www.fhnw.ch/forschung-und-

entwicklung/strategische-

initiativen/alterndegesellschaft).

\section{References}

Bachmann, N., Süsstrunk, S., Bleisch, S., Hollenstein, D., Fabian, C., Huber, T., ... Jäger, J. (2016). Abschlussbericht Projekt „Mit den Augen betagter Frauen, MABF“ SI 1 Alternde Gesellschaft, FHNW. Olten. 
Borgo, R., Kehrer, J., Chung, D. H. S., Maguire, E., Laramee, R. S., Hauser, H., ... Chen, M. (2013). Glyphbased Visualization: Foundations, Design Guidelines, Techniques and Applications. In M. Sbert \& L. Szirmay-Kalos (Eds.), Eurographics 2013, STAR - State of The Art Report. Girona, Spain.

Clifton, K. J., Livi Smith, A. D., Rodriguez, D. (2007). The development and testing of an audit for the pedestrian environment. Landscape and Urban Planning 80 (2007), 95-110.

Hollenstein, D., \& Bleisch, S. (2016). Walkability for different urban granularities. International Archives of the Photogrammetry, Remote Sensing and Spatial Information Sciences - ISPRS Archives, 41(July), 703708. http://doi.org/10.5194/isprsarchives-XLI-B2-7032016

Li, J., Martens, J. B. O. S., \& Wijk, J. J. van. (2010). A model of symbol size discrimination in scatterplots. In Proceedings of the 28th ACM Conference on Human Factors in Computing Systems (CHI 2010) (pp. 2553 2562). Atlanta GA, USA, April 10-15, 2010.

Slocum, T. A., McMaster, R. B., Kessler, F. C., \& Howard, H. H. (2009). Thematic cartography and geovisualization (3rd ed.). Upper Saddle River: Pearson Prentice Hall. 